\title{
The Self-Equalizing De Bruijn Sequence for 3D Profilometry
}

Tomislav Petković

tomislav.petkovic.jr@fer.hr

Tomislav Pribanić

tomislav.pribanic@fer.hr

Matea Đonlić

matea.donlic@fer.hr
University of Zagreb

Faculty of Electrical Engineering and Computing

Unska 3, HR-10000 Zagreb, Croatia
Three-dimensional non-invasive surface profilometry using structured light (SL) is a widely studied topic in the field of computer vision [2]. One of the most important issues of all SL systems is the design of a SL pattern: the pattern must enable reconstruction robust to the ambient light and to the characteristics of the observed object. SL patterns may be classified as one-shot patterns that enable reconstruction from a single image and as multi-shot patterns that require more than one image. Nowadays a well-designed pattern should enable both one and multi-shot reconstruction. Such SL patterns were proposed by Li Zhang et al. [3] for color light and Yueyi Zhang et al. [4] for monochromatic light.

We propose a novel De Bruijn sequence for color or multi-channel SL which we call the self-equalizing sequence. The proposed SL sequence enables a dense multi-shot reconstruction for static objects and a sparse one-shot reconstruction for moving objects. Additionally, the proposed sequence has several desirable properties: (1) it enables the extraction of ambient lighting, (2) it enables the cancellation of object albedo, and (3) it enables the equalization of channel gains. The proposed sequence thus effectively removes the need for a precise color calibration of the imaging system, which is a major advantage in real-world applications.

Current approaches to SL pattern construction based on a De Bruijn sequence either use a complete sequence or impose a constraint on neighboring colors only. We propose to impose the novel self-equalizing constraint on the entire De Bruijn window in such way to achieve the aforementioned desirable properties. The constraint essentially requires that in every De Bruijn window all channels span the full available dynamic range, which is the sufficient condition for the removal of ambient lighting, cancellation of object albedo, and equalization of channel gains thus enabling robust identification of the projected color. A De Bruijn sequence is associated with Eulerian cycle of a De Bruijn digraph which may be used to generate it. The proposed sequence is constructed as Eulerian cycle in the pruned De Bruijn digraph where edges belonging to the invalid windows that do not satisfy the proposed self-equalizing constraint are removed.

The proposed SL pattern is a cyclic sequence of images whose cycle is comprised of $L \cdot S$ frames ( $L$ is sequence length, $S \geq 3$ is the number of phase shifts per symbol), of which any one frame is sufficient for oneshot reconstruction, and of which any $n \cdot S(n \ll L)$ consecutive frames are sufficient for multi-shot reconstruction.

One model for RGB color-space widely accepted in the literature which relates recorded color $I_{c}$ to projected color $I_{p}$ under the influence of object albedo $K$ and ambient lightning $I_{0}$ is proposed by Caspi et al. [1]:

$$
\underbrace{\left[\begin{array}{l}
R \\
G \\
B
\end{array}\right]}_{I_{c}}=\underbrace{\left[\begin{array}{lll}
a_{R R} & a_{R G} & a_{R B} \\
a_{G R} & a_{G G} & a_{G B} \\
a_{B R} & a_{B G} & a_{B B}
\end{array}\right]}_{A} \underbrace{\left[\begin{array}{ccc}
k_{R} & 0 & 0 \\
0 & k_{G} & 0 \\
0 & 0 & k_{B}
\end{array}\right]}_{K} f(\underbrace{\left[\begin{array}{l}
r \\
g \\
b
\end{array}\right]}_{I_{p}})+\underbrace{\left[\begin{array}{l}
R_{0} \\
G_{0} \\
B_{0}
\end{array}\right]}_{I_{0}},
$$

We propose a simplified model where $i$ th channel is modeled as

$$
I_{c}(i)=h(i) I_{p}(i)+I_{0}(i),
$$

i.e. the channel cross-talk is disregarded making $A \cdot K$ a diagonal matrix. The cross-talk may be omitted if the color filters of the projector and the camera are well matched, which holds for standardized consumer electronics and which was also noted by Caspi et al. [1]. Combining the model (2) with the self-equalizing constraints imposed on the De Bruijn sequence allows the recovery of $I_{p}(i)$ from $I_{c}(i)$ independently for each channel.

We have performed three experiments: (1) the influence of channel gains, (2) the reconstruction of white and colored planar surfaces, and (3) qualitative reconstructions of several objects: fruits and vegetables, a
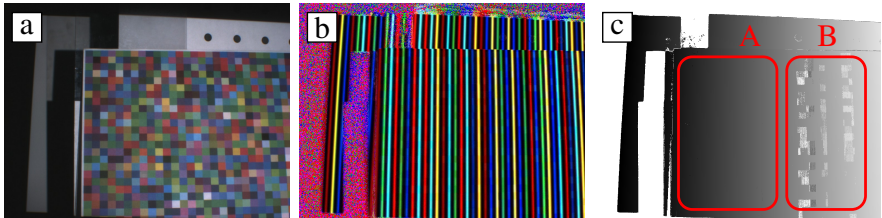

Figure 1: An extremely demanding colorful checkerboard: (a) scene seen by the camera; (b) equalized image; (c) unwrapped phase of the multishot method. Note the unwrapping succeeds for region A and fails for region $\mathrm{B}$ due to cross-talk which is disregarded in the current model.
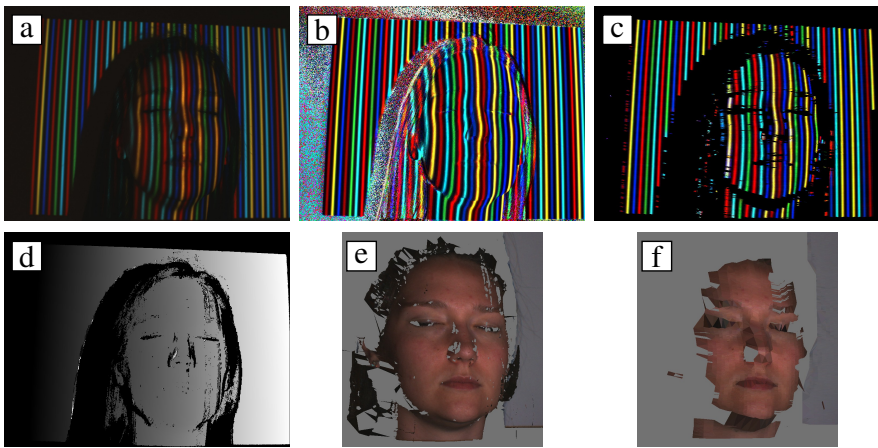

Figure 2: A female face: (a) the first of $n \cdot S$ input images; (b) temporally equalized input image (a); (c) spatially equalized input image (a); (d) the unwrapped phase; (e) textured 3D reconstruction using multi-shot method; (f) textured 3D reconstruction using one-shot method. Note that both the temporal and the spatial equalization successfully remove ambient lightning, object albedo, and varying channel gains.

human face, and a human hand. The first experiment demonstrates the insensitivity to artificially varying channel gains. The reconstruction of a colored planar surface of the second experiment demonstrates the insensitivity to object albedo, but only if there is no significant cross-talk between channels (see Fig. 1). The third experiment illustrates expected results in a real-world application; here we show results for a female face (Fig. 2).

[1] Dalit Caspi, Nahum Kiryati, and Joseph Shamir. Range imaging with adaptive color structured light. Pattern Analysis and Machine Intelligence, IEEE Transactions on, 20(5):470-480, May 1998. ISSN 0162-8828. doi: 10.1109/34.682177.

[2] Joaquim Salvi, Sergio Fernandez, Tomislav Pribanić, and Xavier Lladó. A state of the art in structured light patterns for surface profilometry. Pattern Recognition, 43(8):2666-2680, 2010. doi: 10.1016/j.patcog.2010.03.004.

[3] Li Zhang, Brian Curless, and Steven M. Seitz. Rapid shape acquisition using color structured light and multi-pass dynamic programming. In 3D Data Processing Visualization and Transmission, pages 24-37, 2002. doi: 10.1109/TDPVT.2002.1024035.

[4] Yueyi Zhang, Zhiwei Xiong, Zhe Yang, and Feng Wu. Real-time scalable depth sensing with hybrid structured light illumination. Image Processing, IEEE Transactions on, 23(1):97-109, Jan 2014. ISSN 1057-7149. doi: 10.1109/TIP.2013.2286901. 